CELLULAR CYTOTOXICITY

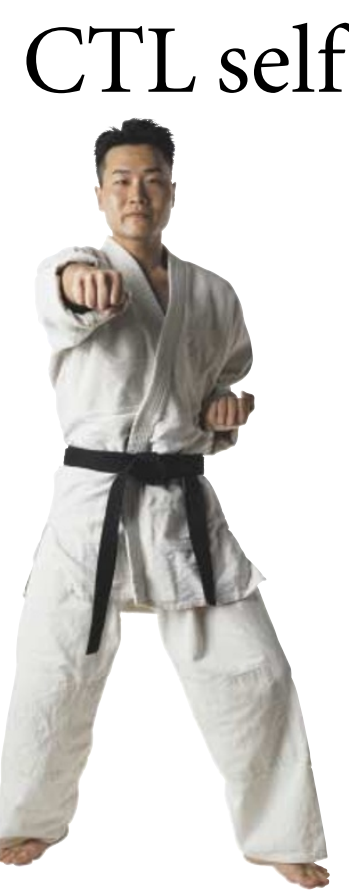

protein perforin, but the molecular basis for this is unknown. The authors of this study reasoned that a granule component would be a good candidate to mediate resistance as it could provide local protection during granule exocytosis. Cytotoxic granules contain perforin, granzymes (serine proteases) and lysosomal enzymes, including thiol cathepsin endoproteases, which can maintain their proteolytic activity in the extracellular environment. Before the perforin pore-forming complex is assembled, perforin passes through an intermediate membrane-associated stage that is highly susceptible to proteolysis. So, does a cathepsin that is expressed on the cytotoxic-cell surface after exocytosis cleave perforin and, in so doing, provide protection for effector cells?

To investigate this, Balaji et al. treated CTLs cultured on plate- bound anti-CD3 antibodies (to trigger degranulation) with cathepsin inhibitors. These drugs resulted in rapid T-cell suicide, which implies that cathepsins do participate in CTL self-protection. This CTL suicide does not require the Fas-FasL death pathway, as it occurred normally in CTLs from gld (FasLmutant) mice, but it does require perforin, as it did not occur in CTLs from perforin-knockout mice.

Further experiments with membrane-impermeant cathepsin inhibitors and cathepsin-B-specific inhibitors showed that cathepsinmediated protection of degranulating CTLs against perforin attack occurs in an extracellular location and that cathepsin B is required specifically. The authors also showed that CTLs express little surface cathepsin B before degranulation, but after T-cell-receptor triggering, the surface expression of this

\section{NUCLEAR TRANSPORT}

\section{Two-way traffic}

The Smad signalling pathway functions downstream of transforming growth factor (TGF)- $\beta$ receptors. Receptor-regulated Smad proteins, Smad 2 and Smad 3 , are phosphorylated after TGF- $\beta$ stimulation, then each forms a complex with Smad4 and translocates to the nucleus. There, together with DNA-binding cofactors, they direct transcriptional activity. Now, the groups of Joan Massagué and Caroline Hill report new insights into the transport of Smads in and out of the nucleus.

Massagué and colleagues focused on Smad2 and showed that it binds directly to nucleoporins CAN/Nup214 and Nup153, which are located on the cytoplasmic and nuclear side of the nuclear pore complex, respectively. They could inhibit Smad2 nuclear import by adding an excess of a CAN/Nup214 fragment that contains the Smad2-binding domain, as well as by using Smad2 mutants with reduced affinity for CAN/Nup214. This indicated to the authors that direct contact with CAN/Nup214 is needed for Smad2 nuclear import.

On the other hand, both nucleoporins might be required for nuclear export, because Smad 2 mutants defective in Nup153 and/or CAN/Nup214 binding inhibited this process. The authors propose that CAN/Nup214 and Nup153 act as docking sites for nuclear import and export, respectively.

Using a nucleocytoplasmic shuttling assay, Massagué and co-workers also showed that Smad DNA-binding factor FAST-1 and cytoplasmic retention factor SARA compete with the nucleoporins for Smad 2 binding and therefore inhibit nucleocytoplasmic shuttling. TGF- $\beta$ signalling also inhibits nucleocytoplasmic shuttling, which can be explained by the finding that Smad2 phosphorylation causes a decrease in its affinity for SARA and an increase in its affinity for Smad4. The authors propose that, in unstimulated cells, Smad2 undergoes continuous nucleocytoplasmic shuttling, and that TGF- $\beta$-induced Smad 2 phosphorylation causes retention of Smad 2 in the nucleus by decreasing its affinity for SARA in favour of Smad4.

Hill and colleagues complemented this work by asking what happens to endogenous Smads during active TGF- $\beta$ signalling. Using an inhibitor that specifically blocks continuous TGF- $\beta$ receptor signalling, they showed that receptors remain active for at least 3-4 hours after TGF- $\beta$ stimulation, and that continuous receptor activity is necessary for optimal transcription. However, accumulation of phosphorylated Smad2 and 3 in the nucleus is complete in 30 minutes. So, how do the active receptors influence Smads once they are in the nucleus? And why is continuous receptor activity needed?

To address the first question, the authors used immunofluorescence and showed that after TGF- $\beta$ stimulation, rather than remaining statically in the nucleus, Smads cycle continuously between the cytoplasm and the nucleus. The requirement for continuous receptor activity became obvious when Hill and co-workers found that Smad 2 and 3 are dephosphorylated and dissociated from Smad 4 before returning to the cytoplasm. In the cytoplasm, Smad 2 and 3 are re-phosphorylated by the active receptors, form complexes with Smad4 and re-enter the nucleus. So, the shuttling capacity of Smad 2 and 3 allows continuous monitoring of receptor activity by the signaltransducing Smads.

The model that Hill proposes features a prominent role for a nuclear phosphatase that allows nuclear exit of Smad2 and 3. The race is now on to identify this nuclear phosphatase.

Arianne Heinrichs

(D) References and links ORIGINAL RESEARCH PAPERS $X u$, L., Kang, Y., Çöl, S. \& Massagué, J. Smad2 nucleocytoplasmic shuttling by nucleoporins CAN/Nup214 and Nup153 feeds TGF- $\beta$ signaling complexes in the cytoplasm and nucleus. MOl. Cell 10, 271-282 (2002) | Inman, G. J., Nicolás, F. J. \& Hill, C. S. Nucleocytoplasmic shuttling of Smads 2, 3, and 4 permits sensing of TGF- $\beta$ receptor activity. Mol. Cell 10, 283-294 (2002) 
potentially protective enzyme increases rapidly. Finally, CTL surface cathepsin B was shown to be enzymatically active and to cleave perforin efficiently.

This work indicates that cytotoxic lymphocytes release granule-derived cathepsin B on degranulation, which associates with the cell surface and protects these cells against perforin attack and self-destruction. So, a long-standing question about the granule-exocytosis model seems to have been answered.

Jenny Buckland, Associate Editor, Nature Reviews Immunology

\section{6) References and links}

ORIGINAL RESEARCH PAPER Balaji, K. et al.

Surface cathepsin B protects cytotoxic

lymphocytes from self-destruction after

degranulation. J. Exp. Med. 196, 493-503

(2002)

FURTHER READING Barry, M. \& Bleackley, R. C. Cytotoxic T lymphocytes: all roads lead to death. Nature Rev. Immunol. 2, 401-409 (2002)

Pierre Henkart's lab:

http://www3.cancer.gov/intra/EIB/henkart.htm

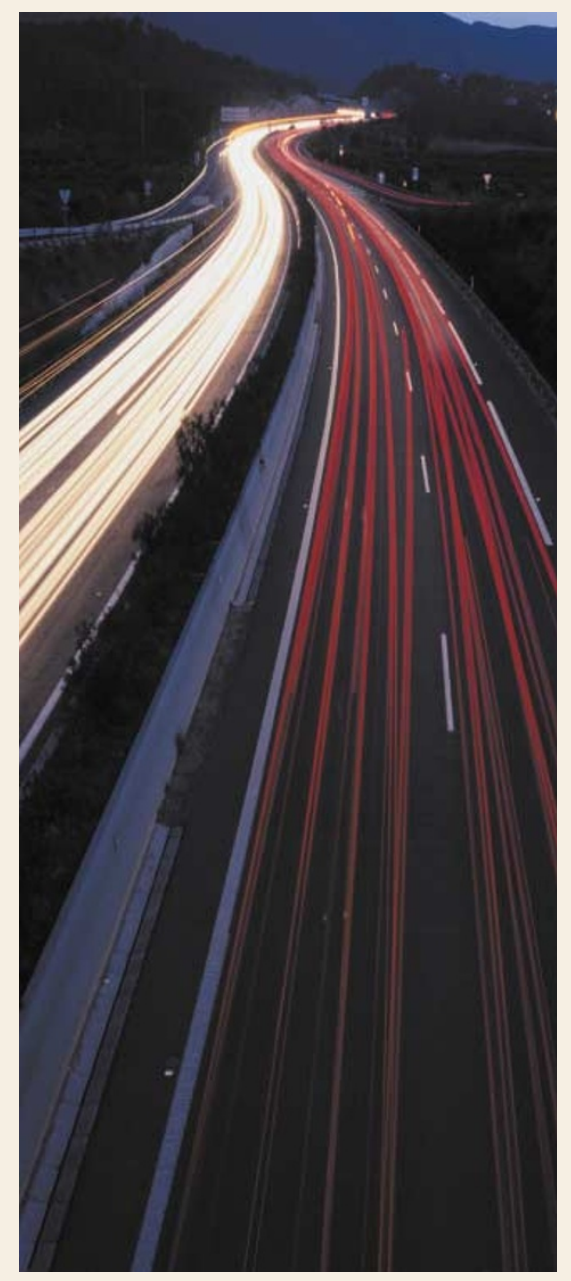

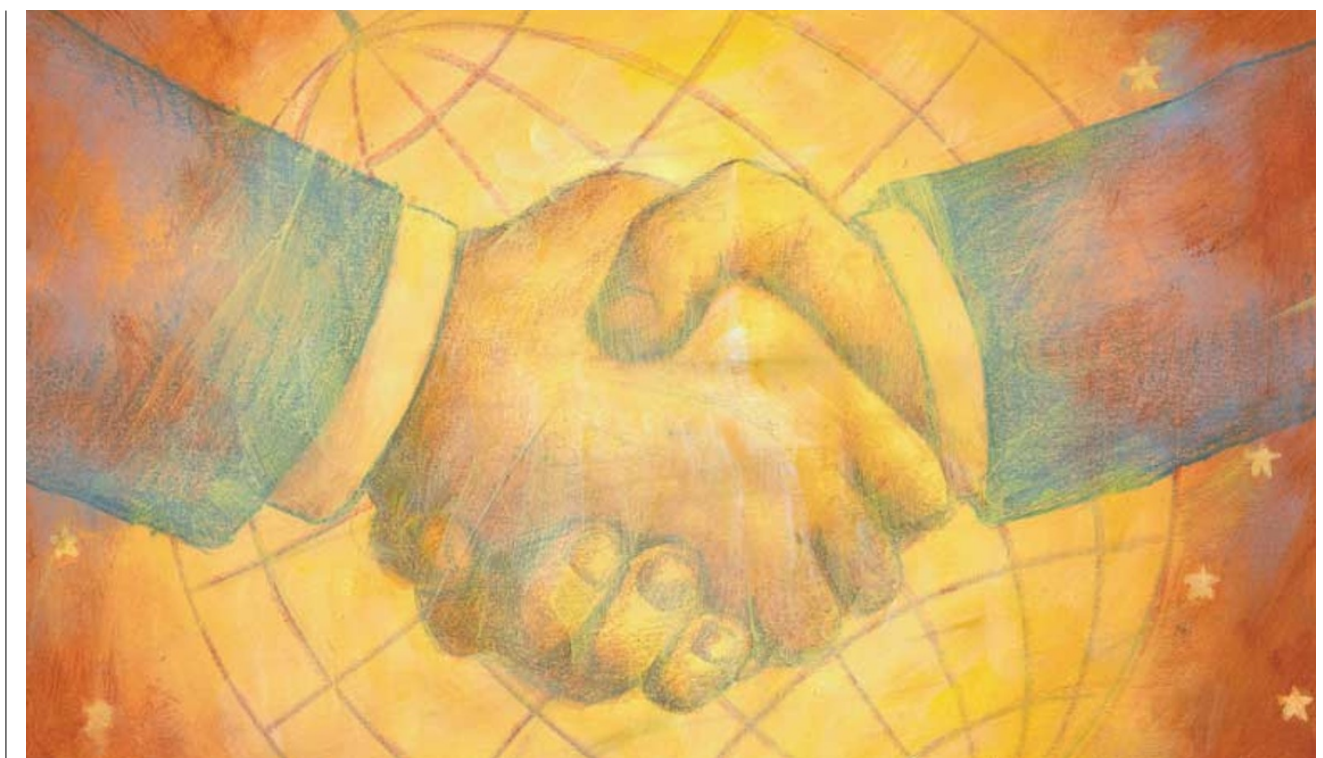

CELL ADHESION

\section{Promoting partnerships}

Some areas of cell biology, despite a wealth of data, remain confusing. So far, the role of Rac in adhesion has been one such area, but James Nelson's group now propose a function for Rac in the kinetics and strengthening of E-cadherin-mediated cell-cell adhesion.

To study membrane activity dynamically at cell-cell contacts, Nelson's group used time-lapse phase-contrast microscopy, and saw that exploratory lamellipodia were responsible for the initial contacts that were made between MDCK cells. Once opportunistic collisions between cells had occurred, a local burst of lamellipodia was seen at the intercellular contact sites.

The small Rho GTPase Rac regulates the formation of lamellipodia, so, to be sure of Rac's involvement, the authors used green fluorescent protein (GFP)-tagged Rac1. GFP-Rac1 localized to the tips of extending lamellipodia in migrating cells, but once cell-cell contact had been made, it accumulated at the newly contacting membranes. Over time, though, as the contact area expanded, lamellipodia formation decreased, as did the intensity of the GFP-Racl signal at the original cell-cell contact site. Concomitant with the increase in contact area, the authors noticed a relative decrease in the number of lamellipodia that were originally present around the cell periphery.

As is common when studying small GTPases, dominant-negative and constitutively active constructs were used. Cell-cell contacts in cells that expressed dominant-negative (T17N) Rac grew very slowly. And not only did fewer lamellipodia form, but the ones that did form weren't restricted to cell-cell contacts alone, so the 'relative protrusive index' decreased. By contrast, constitutively active Rac induced increased lamellipodia formation. As neither construct prevented E-cadherin accumulation at cell-cell contacts, the changes in intercellular adhesion seemed to result from Rac1-dependent actin-based membrane protrusions rather than Ecadherin distribution. But Nelson and colleagues raised the possibility that, by increasing lamellipodia formation, the resultant increase in surface area between the contacting membranes probably increases the likelihood of E-cadherin-mediated cell-cell adhesion.

Finally, Nelson's group analysed Rac's role in the strength of adhesion using a 'hanging drop' cell-cell adhesion assay. Here, cells are forced together, and the sizes of cell aggregates and their resistance to trituration (breaking up of cell clumps) are measured. Consistent with impaired cell-cell contact formation, T17NRac cell aggregates were weaker, and developed resistance to trituration more slowly than their wild-type counterparts. But, despite the altered kinetics and strength of cell-cell adhesion, intercellular contacts did eventually form, indicating that Rac's primary function, through lamellipodia, might be to increase the rate of contact formation and strengthening.

(2) References and links

Katrin Bussell

ORIGINAL RESEARCH PAPER Ehrlich, J. S., Hansen, M. D. H. \& Nelson, W. J. Spatio-temporal regulation of Rac1 localization and lamellipodia dynamics during epithelial cell-cell adhesion. Dev. Cell $\mathbf{3}$, 259-270 (2002)

FURTHER READING Fukata, M. \& Kaibuchi, K. Rho-family GTPases in cadherin-mediated cell-cell adhesion. Nature Rev. Mol. Cell Biol. 2. 887-897 (2001) 